\title{
Private Practitioners Knowledge of Local Anaesthetic Systemic Toxicity (LAST)
}

\author{
Neha Yadav르, Reshu Madan Sanan², Shefali Phogat³ ${ }^{3}$ Nisha Yadav, Nupur Dabas ${ }^{5}$, Pankaj Ritwal ${ }^{6}$ \\ 1, 2,3,4,5,6 Department of Prosthodontics, Faculty of Dental Sciences, SGT University, Gurgaon, Haryana India.
}

\section{ABSTRACT}

\section{BACKGROUND}

Local anaesthetics (LAs) by definition are drugs that reversibly block the transmission of a nerve impulse, causing reversible absence of pain sensations without affecting consciousness. As a complication of local anaesthetic administration, a condition called as local anaesthetic systemic toxicity (LAST) can occur which affects central nervous system (CNS) and cardiovascular system (CVS). Local anaesthetic systemic toxicity is a very serious condition that can cause death. In LAST, blood levels of anaesthetic agent exceed the maximum dose, usually as a result of multiple injections or an accidental injection into a vein. The main objective of this survey was to assess the knowledge of LAST among private practitioners and to spread awareness about this common but often neglected complication of local anaesthetic administration.

\section{METHODS}

A survey was conducted with a questionnaire comprising of 15 questions for 251 private practitioners (registered in Indian Dental Association, Gurugram). The survey was mailed to the participants and the response was collected through online mode only. Using Excel program, the results were organized in graphs and tables, and presented as descriptive statistics.

\section{RESULTS}

Of the 251 surveys sent online, 151 were completed, with a survey completion rate of $60 \%$. On an average, only $39.9 \%$ of the participating dentists had adequate knowledge of local anaesthetic systemic toxicity. Based on the survey, only $3.3 \%$ of participating dentists knew how to use lipid treatment and $55.6 \%$ had no idea about lipid treatment.

\section{CONCLUSIONS}

If LAST occurs, the key is to recognize it immediately and institute appropriate management. Based on the survey, there is a need to create awareness about local anaesthetic systemic toxicity and its management among dentists.

\section{KEY WORDS}

Local Anaesthesia, Last, Complication, Toxicity, Overdose
Corresponding Author: Dr. Reshu Madan Sanan, Department of Prosthodontics, SGT University, Gurgaon, Haryana India. E-mail: reshu.sanan@sgtuniversity.org

DOI: $10.14260 / \mathrm{jemds} / 2021 / 724$

How to Cite This Article: Yadav N, Sanan RM, Phogat S, et al. Private practitioners knowledge of local anaesthetic systemic toxicity (LAST). J Evolution Med Dent Sci 2021;10(41):35713576, DOI: 10.14260/jemds/2021/724

Submission 25-03-2021,

Peer Review 29-05-2021,

Acceptance 05-06-2021,

Published 11-10-2021.

Copyright (C) 2021 Neha Yadav et al. This is an open access article distributed under Creative Commons Attribution License [Attribution 4.0 International (CC BY 4.0)] 


\section{BACKGROUND}

Local anaesthetics by definition are drugs that reversibly block the transmission of a nerve impulse, causing reversible absence of pain sensations without affecting consciousness. Local anaesthetic is used by dentists frequently in almost every minor and major dental procedure and proper knowledge about various LAs, their complication and their management is of utmost importance. In India, dentists are trained for local anaesthesia in the beginning of their undergraduation training. They are taught about various LAs, their use, complications and management both theoretically and practically.

Doctors first began to use local anaesthetic agents, including cocaine, in the 1860s. Seizures, adverse cardiac effects and respiratory failure were often associated with their use. The development of newer, less toxic amino esters began with procaine in 1905. However, amino esters had slow onset and high allergic potential, leading to the development of the amide-type anaesthetics lignocaine in 1943. Several other amide-type anaesthetics have been developed for use specifically in dentistry: prilocaine, bupivacaine, articaine, etidocaine and mepivacaine. ${ }^{1}$

As a complication of local anaesthetic administration, a condition called as local anaesthetic systemic toxicity can occur which effects central nervous system and cardiovascular system. ${ }^{2}$ Local anaesthetic systemic toxicity is a very serious condition that can cause death. In LAST, blood levels of anaesthetic agent exceed the maximum dose, usually as a result of multiple injections or an accidental injection into a vein. If the level of LA in the blood reaches an individual's maximum dose, LAST may occur. Weinberg et al. noted bradycardia and then serious ventricular arrhythmia in a patient who had received $22 \mathrm{mg}$ of bupivacaine. They found a serious carnitine defect and isovaleric acidaemia in this patient. ${ }^{3}$ Prevention is understandably the most vital element in avoiding morbidity and mortality rates associated with local anaesthetic systemic toxicity, but despite best practices, cases still occur. It is important to have knowledge about how to manage these uncommon events.

After administration of LA, any abnormal changes related to cardiovascular or neurological system should raise concern of LAST. The signs and symptoms of LAST may vary and importantly cardiovascular collapse may occur even without precursory neurological changes. After the initial LA administration, the practitioner should be attentive as LAST can occur anytime even when the patient is less supervised. An initial excitatory state followed by a depressive phase is classically a two-staged process of central nervous system toxicity. Early signs related to neurological system include tinnitus, slurred speech and tremor. There can also be a change in mental status of the patient. Without these factors also, LAST can occur. The neurological phase results in generalized convulsions which can lead to a respiratory depression or coma.

Cardiovascular system toxicity occurs in 3 phases. First is the phase of hypertension and tachycardia. The second is an intermediate phase which is associated with myocardial depression and hypotension. In terminal (last) phase, variety of arrhythmias and a systole occurs. But these symptoms may lead to differential diagnosis and subsequent treatment in absence of the knowledge of systemic toxicity.
In 1998 and 2000, researchers conducted several animal studies investigating the treatment of LAST with lipids. ${ }^{4,5}$ In 2006, Rosenblatt et al. reported a case in which bupivacaine and mepivacaine were administered as brachial plexus blocks, inducing cardiac arrest. Resuscitation was attempted for 20 minutes without response. The hemodynamic parameters started returning to normal when an IV injection of a lipid emulsion of $100 \mathrm{ml}$ of $20 \%$ intra lipid (Fresenius Kabi; Uppsala, Sweden) was given. ${ }^{6}$ The work done in the year 2007 in UK and year 2008 in US, further advanced the IV lipid therapy. Since then, lipid therapy has come in the cardiac arrest resuscitation guidelines for LAST cases. ${ }^{7}$ The American Society of Regional Anaesthesia and Pain Medicine in 2010, noted the importance of cardiopulmonary resuscitation (CPR) and intra lipid therapy in cases of cardiac arrest induced by LAST. ${ }^{8}$ The same year, the American Heart Association guidelines for LAST-induced cardiac arrest included lipid therapy in the section of the algorithms for advanced cardiac life support guidelines on special situations. ${ }^{9}$

Though extensive guidelines exist for management of LAST, the precursor knowledge of this life-threatening event is not commonly observed or taught. The main objective of this survey was to assess the knowledge of LAST among private practitioners and to spread awareness about this common but often neglected complication of local anaesthetic administration.

\section{METHODS}

The questionnaire was developed using the guidelines given by Eysenbach, that is, the checklist for reporting results of internet E-surveys (CHERRIES). This study was conducted from June 2020 to August 2020. Following this, the validation analyses were performed, namely, face validation to ensure understanding and to minimise response bias, pilot data preparation. The questionnaire was well understood by the respondents. Content was validated as per the aim of the study. Checking the relevance of the questions was the last step under content reliability.

A questionnaire comprising of 15 questions was provided to 251 private practitioners registered and practicing in Gurugram, Haryana, India. The survey was mailed to the participants and response was collected within one week through online mode only. It was conducted from June 2020 to August 2020. Questionnaire consisted of 15 questions; few questions were based on general questions regarding local anaesthesia. The other few questions were based on local anaesthetic systemic toxicity, its signs and symptoms, and its management. Questions regarding lipid emulsion therapy were also asked. Dentists were also asked if they have lipid solution in their clinic or not and if they knew how to or when to use lipid therapy. A complete correct answer was assigned 5 point and an incorrect/unknown answer was assigned 0 points. The total knowledge score ranged from 0 to 60 , with a higher score denoting a better knowledge. The Cronbach's alpha coefficient of the knowledge questionnaire was 0.67 in present research, indicating acceptable internal consistency. The three questions (q1, q5, q6) were assigned 1 point, if the response selected was "yes", with a score range of $0-15$. The total score range was 0 to 75 for the present questionnaire. 


\section{Statistical Analysis}

All the online responses were entered into electronic data base. Data was analysed using Statistical Package for Social Sciences (SPSS) version 21, IBM Inc. Summarized data was presented using tables and graphs. Data was not normally distributed as tested using the Shaperio-Wilk W test (P value was less than 0.05). Kruskal-Wallis was used for comparison of two or more groups. Mann-Whitney $U$ test was used for two groups. Level of significance was set at $\mathrm{P}<0.05$.
If the 251 surveys sent online, 151 were completed, with a survey completion rate of $60 \%$. The knowledge and practice scores among study participants based on their educational qualification were compared using Kruskal-Wallis test. It was found to be significant $(\mathrm{P}=0.039)$ with maximum knowledge and practice scores being reported by oral surgeons, followed by endodontists, then prosthodontists, oral medicine and radiologists, oral pathologists, paedodontists, periodontists, orthodontists, general dentists and least in public health dentists.

\section{RESULTS}

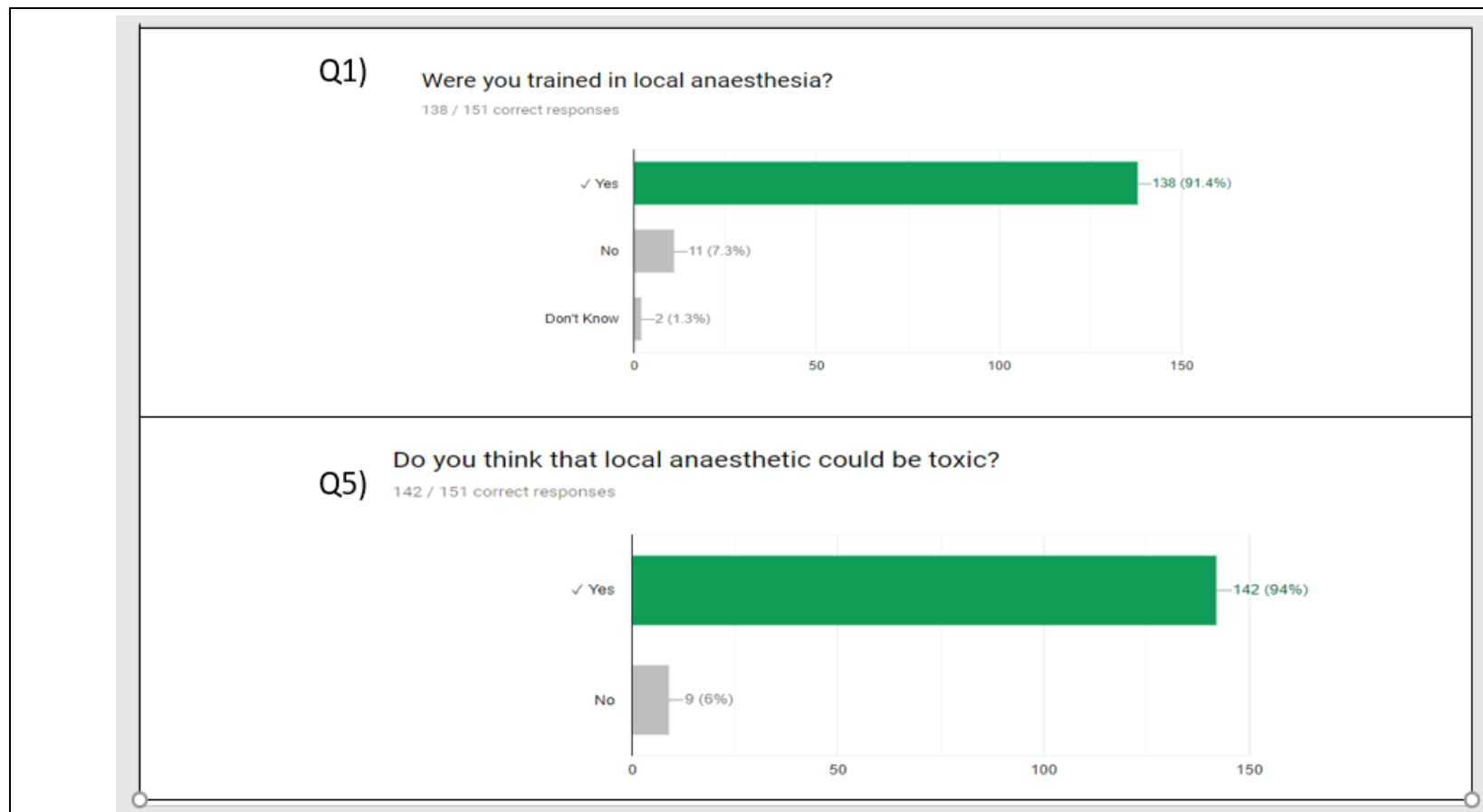

Figure 1. In the Above Figure, Few General Questions Regarding Local Anaesthesia Were Asked and the Results are Shown Graphically

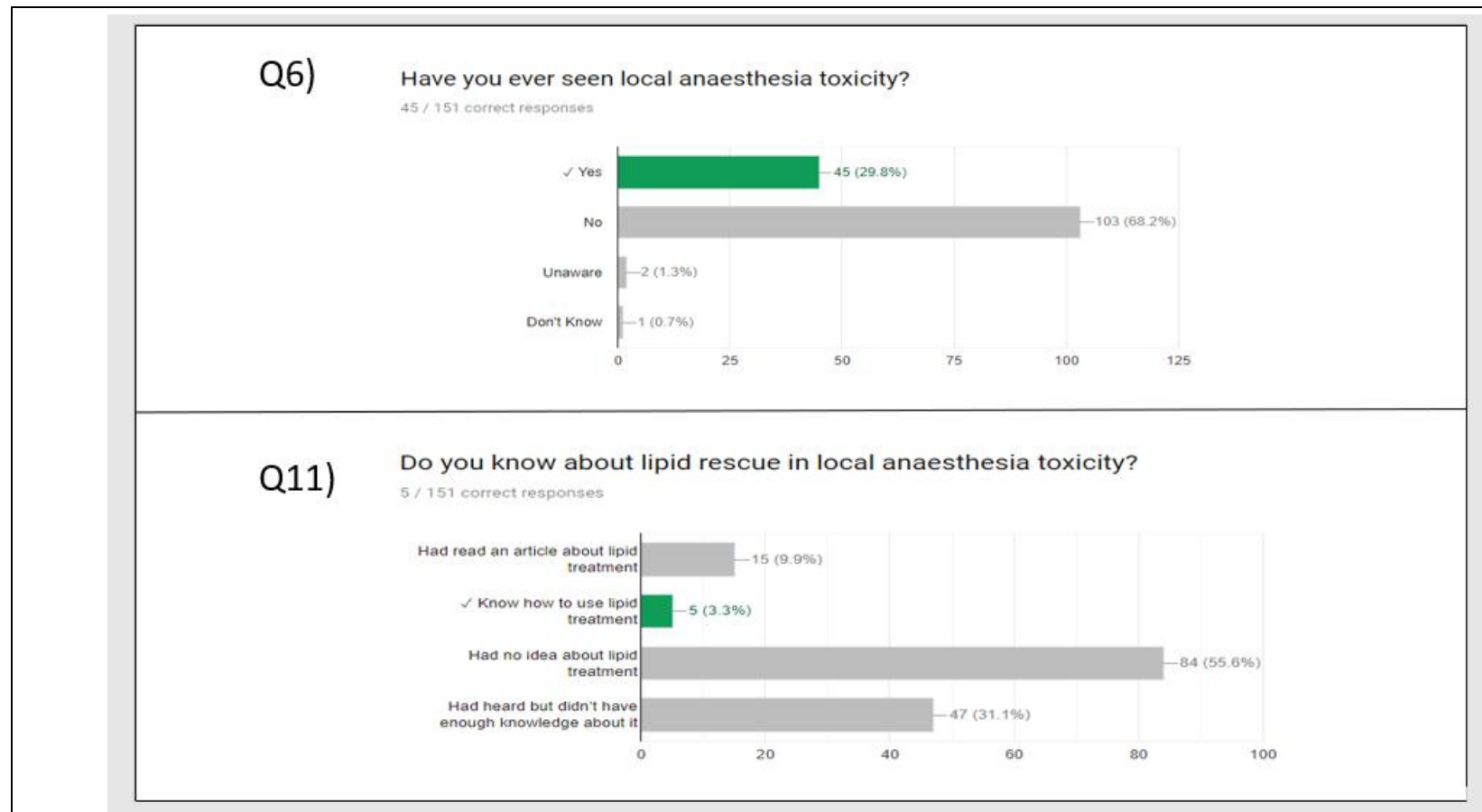

Figure 2. In this Figure, There is Graphical Representation of the Results for the Questions Asked about Local Anaesthetic Systemic Toxicity (LAST) 


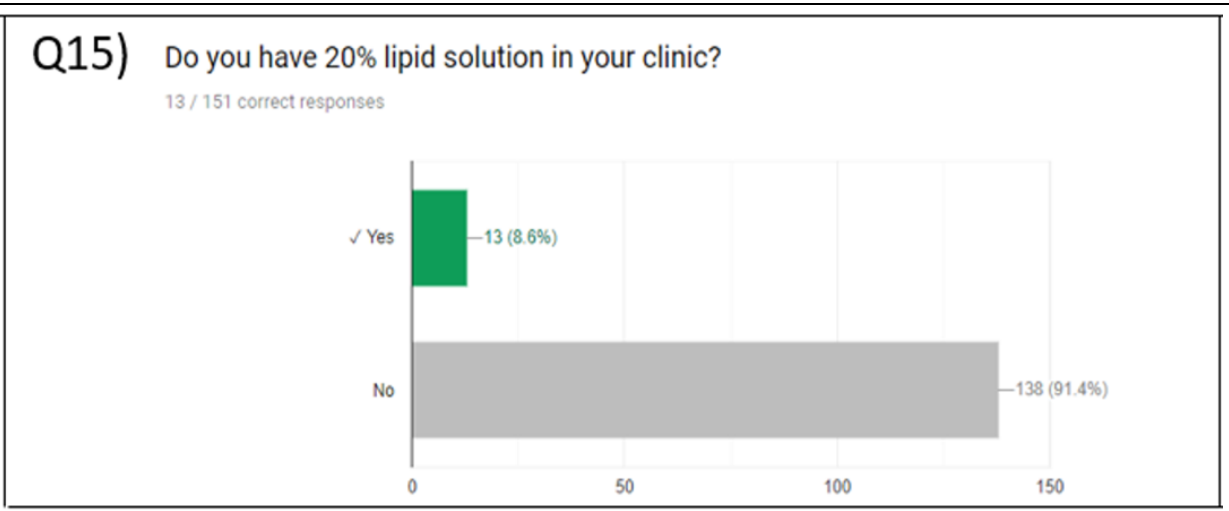

Figure 3. This Figure is Also a Graphical Representation of the Questions Regarding LAST and its Management Including LIPID Emulsion Therapy

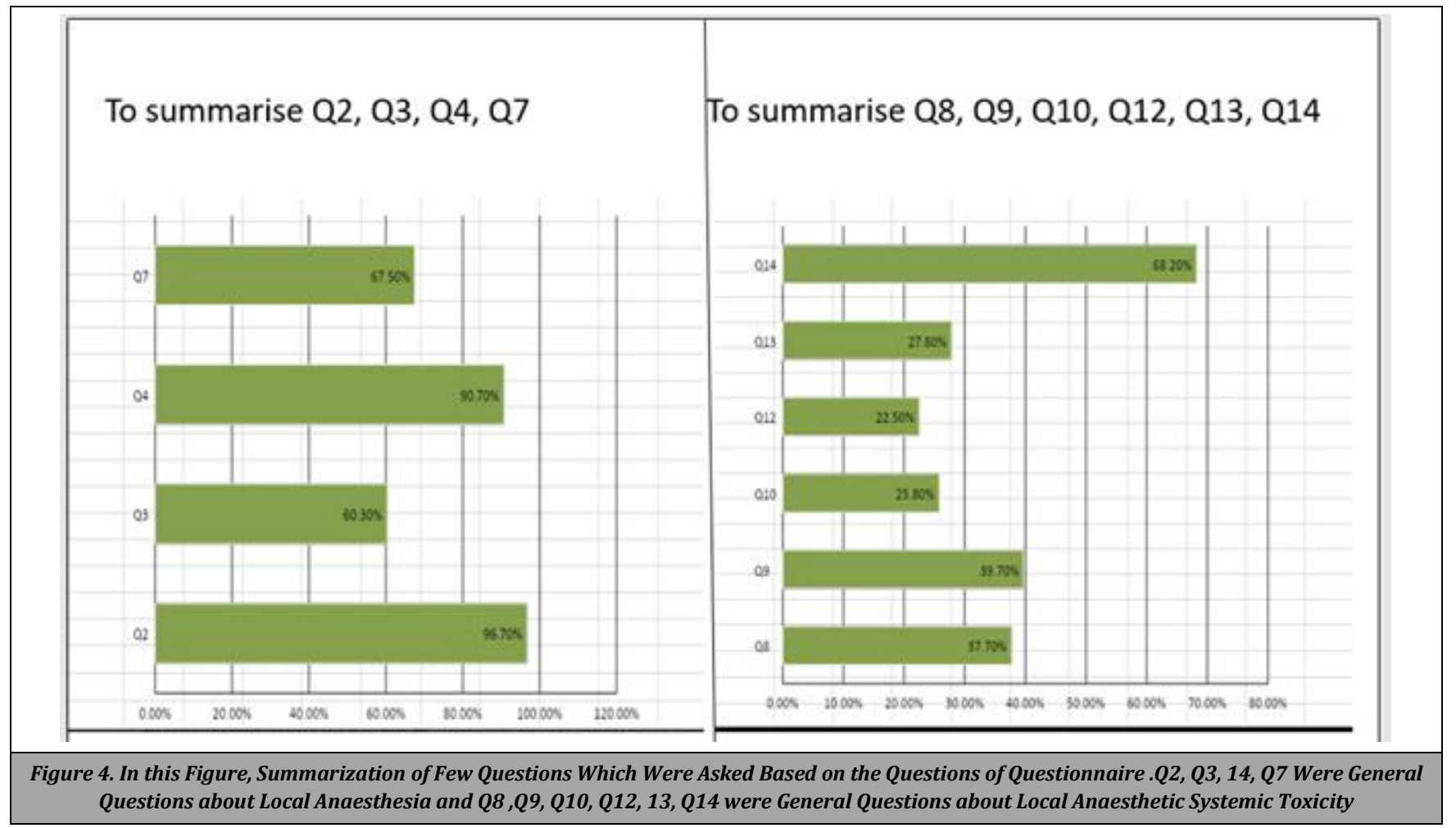

\section{DISCUSSION}

On pair wise comparison using Mann-Whitney U test, significant differences for the knowledge and practice scores were seen between oral surgeons to that of paedodontists, periodontists, orthodontists, general dentists and public health dentists.

The knowledge and practice scores among study participants based on their years of experience were compared using Kruskal-Wallis test. It was not found to be significant. Although maximum scores were reported by the study participants who had an experience of 14 years and above.

Knowledge of local anaesthesia is of utmost importance for a dentist for various surgical and non-surgical procedures. $96.7 \%$ participating dentists were aware that LA blocks pain sensation without loss of consciousness. $60.3 \%$ people were aware that bupivacaine does not have the shortest half-life. $90.7 \%$ people knew that sodium channels are the main target for activity of LA. $67.5 \%$ people were aware that out of the options given, injection site vascularity determines extent of systemic LA absorption. It was calculated that on an average, $78.8 \%$ of participating dentists had basic knowledge of LA.

$6 \%$ of participating dentist were unaware of the fact that local anaesthesia is toxic. Around one third of the participating dentists i.e. $29.8 \%$ had seen LA toxicity in one form or the other. This percentage supports the fact that LA toxicity is quite common. $37.7 \%$ of participating dentists were aware of the fact that local anaesthetic systemic toxicity may be seen in any patient receiving a regional block. Only $25.8 \%$ of participating dentists knew exact signs and symptoms of LAST. $22.5 \%$ of participating dentists were aware about the treatment of LAST, i.e. rapid administration of $20 \%$ lipid emulsion. $68.2 \%$ of participating dentists were aware about the correct dose of $20 \%$ lipid emulsion therapy. On an average, only $39.9 \%$ of the participating dentists had adequate knowledge of local anaesthetic systemic toxicity. Based on the survey, only $3.3 \%$ of participating dentists knew how to use lipid treatment and $55.6 \%$ had no idea about lipid treatment. $91.4 \%$ of participating dentists do not have $20 \%$ lipid solution in their clinic. 


\begin{tabular}{|c|c|c|c|c|c|c|c|}
\hline & Specialization & $\mathbf{N}$ & Mean & SD & $\begin{array}{c}\text { Chi Square } \\
\text { Value }\end{array}$ & $\begin{array}{c}P \\
\text { Value }\end{array}$ & $\begin{array}{l}\text { Post } \\
\text { HOCb }\end{array}$ \\
\hline 1 & General dentist & 8 & 36.88 & 5.30 & \multirow{10}{*}{17.696} & \multirow{10}{*}{$\begin{array}{l}0.039^{*}, \\
\text { SIG }\end{array}$} & \multirow{10}{*}{$>5,8,6,1,9$} \\
\hline 2 & $\begin{array}{l}\text { Oral medicine and } \\
\text { radiologist }\end{array}$ & 10 & 39.00 & 7.38 & & & \\
\hline 3 & Prosthodontist & 26 & 39.62 & 9.99 & & & \\
\hline 4 & Oral surgeon & 17 & 44.71 & 8.92 & & & \\
\hline 5 & Paedodontist & 12 & 37.92 & 6.56 & & & \\
\hline 6 & Orthodontist & 26 & 37.50 & 9.82 & & & \\
\hline 7 & Endodontist & 22 & 41.59 & 7.62 & & & \\
\hline 8 & Periodontist & 15 & 37.67 & 8.63 & & & \\
\hline 9 & Public health dentist & 8 & 31.25 & 6.41 & & & \\
\hline 10 & Oral pathologist & 6 & 38.33 & 12.91 & & & \\
\hline \multicolumn{8}{|c|}{$\begin{array}{l}\text { Comparison of Scores among the Study Participants Based on their } \\
\text { Educational Qualification }\end{array}$} \\
\hline \multicolumn{8}{|c|}{$\begin{array}{l}\text { Kruskal-Wallis test, }{ }^{\text {b }} \text { Mann-Whitney } \mathrm{U} \text { test, Level of significance set at } \mathrm{P}<0.05 \\
\text { *statistically significant } \\
\text { NS: NON-SIGNIFICANT } \\
\text { SIG: SIGNIFCANT }\end{array}$} \\
\hline
\end{tabular}

\section{DISCUSSION}

The survey shows local anaesthetic toxicity is quite common and a large percentage of practicing dentists are not aware of its symptoms or management. Local anaesthesia is used by dentists on a daily basis. The systemic toxicity that it causes is a very serious condition and can lead to death.

A life-threatening event occurs related to local anaesthetic systemic toxicity, after administration of local anaesthetic drugs through various routes. The mechanisms of LAST are multifactorial, mainly effecting central nervous system and cardiovascular system. Understanding of LAST is highly relevant considering the use of LA in various dental procedures. LAST is reported in $0.03 \%$ per 1000 peripheral nerve blocks (according to NCBI), but can be fatal.

Absent or reduced sensory perception around the tongue, metallic taste, light-headedness, dizziness, visual and auditory disturbances (difficulty focusing and tinnitus), disorientation and drowsiness are the general signs and symptoms of local anaesthetic systemic toxicity.

When the plasma concentrations of local anaesthesia increases, initially, the cortical inhibitory pathways are compromised by blocking of $\mathrm{Na}$ channels and subsequently inhibitory neuron depolarization is disrupted. All of this leads to clinical signs and symptoms such as sensory and visual changes, muscular activation, followed by sudden, uncontrolled electrical disturbance in the brain usually called as seizure. So, basically rise in plasma concentration of local anaesthesia affects the excitatory pathways thereby causing a depressive phase of toxicity along with consciousness loss, followed by coma and at last respiratory failure.

Toxic features in the cardiovascular system produced by local anaesthetic toxicity include rhythm disturbances and secondarily myocardial dysfunction. Blockade of sodium channel at bundle of his disrupts normal conduction leading to extended intervals of PQ, QRS and ST wave. Following this reentrant brady arrhythmias, tachyarrhythmias take place, this can be worsened by further blockade of potassium channel which again prolongs the QT interval.

$\mathrm{Na}^{+}-\mathrm{Ca}^{2+}$ exchange pump and calcium channel are various contributory mechanisms for myocardial dysfunction whose blockade causes reduction in calcium stores (intracellularly) which decreases contractility. ${ }^{10}$

Oxygen, standard monitoring and intravenous access should be applied to patients receiving injection of local anaesthesia in doses which are sufficient to cause local anaesthetic systemic toxicity. As delayed presentations are increasingly occurring, monitoring should continue for at least 30 minutes after the LA is given. Immediate access to a LAST management checklist is advisable in the form of a "LAST Rescue Kit" which should always be available while giving LA as it contains all medications and resuscitation equipment.

General safety and resuscitation measures are imperative in any emergency included in prompt management. The very first step is to stop further administration of local anaesthetic call for emergency help. Airway, breathing, and circulation are to be managed immediately on priority basis.

Airway management is essential to obviate the factors which potentiates LAST such as hypoxia, hypercapnia, and acidosis (metabolic or respiratory). After securing airway, administer $100 \%$ oxygen. (Hyperventilation and respiratory alkalosis should also be kept in mind as they can deteriorate the condition.)

Intravenous lipid emulsion therapy has promising results in management of local anaesthetic systemic toxicity post maintenance of airway, breathing and circulation. Various data suggests that intravenous lipid emulsion therapy may ply any LA agent from heart or brain (which are high blood flow organs) and muscles or liver (which are storage or detoxification organs. Lipid therapy can also ameliorate the blood pressure and cardiac output and also facilitates myocardial protection. The data demonstrating the clinical efficacy of lipid emulsion is not available because of difficulties in valid data collection and the bounded feasibility of prospective studies. But various animal studies are present which support lipid emulsion therapy in decreasing mortality when used with proper resuscitative measures. When local anaesthetic systemic toxicity occurs, right after airway management, administer $20 \%$ intravenous lipid emulsion therapy in cases which are potentially serious.

The steps involve administration of a commencing bolus of $100 \mathrm{~mL}$ over 2 - 3 minutes (depending on body weight, if lean body weight is $<70 \mathrm{~kg}, 1.5 \mathrm{mLkg}^{-1}$ is given. This is followed by a $20 \%$ lipid emulsion infusion of $200-250 \mathrm{~mL}$ over $15-20$ minutes (depending on body weight, if the lean body weight is $<70 \mathrm{~kg}, 0.25 \mathrm{~mL} \cdot \mathrm{kg}^{-1} \cdot \mathrm{min}^{-1}$ is given). If the seizure activity intensifies causing metabolic acidosis, prompt prevention and its termination is important. The first line drugs are benzodiazepines. In cases of cardiovascular compromise, Propofol should be used (but in small doses because large doses depress cardiac function). If seizures still continue, low dose neuromuscular blockade can be expected. If cardiac arrest occurs, cardiopulmonary resuscitation (CPR) must be followed. Chest compressions should be started immediately and should be continued till circulation is secured.

In the absence of rapid recovery following advanced life support measures and intravenous lipid emulsion therapy, early rumination should be given to cardiopulmonary bypass for circulatory support. Lipid emulsion therapy effect (inotropic effect) only appears when the myocardial LA levels are below a threshold that is similar to ion channel blocking concentration. Hence accentuates significance of effective chest compressions to certify coronary perfusion, that is sufficient to reduce LA tissue levels in order to obtain the positive benefit of lipid emulsion therapy.

Every dental clinic should have provisions to monitor such patients 2 - 6 hours ( 2 hours in case of CNS symptoms and 6 
hours in case of CVS) post the episode of toxicity. If the symptoms subside, such a patient may be discharged after 6 hours. Follow up calls are important to confirm re-occurrence of symptoms.

\section{CONCLUSIONS}

Within the limitations of this survey, it is concluded that there is a need to create awareness about local anaesthetic systemic toxicity and its management among dentists. Episodes of LAST are usually uncertain, but many are preventable. It is the duty of dentists to make every effort to avoid this emergency. If LAST occurs, the solution is to recognize it instantly and institute proper management. Significantly advanced management of this emergency can be done by lipid emulsion therapy.

Data sharing statement provided by the authors is available with the full text of this article at jemds.com.

Financial or other competing interests: None.

Disclosure forms provided by the authors are available with the full text of this article at jemds.com.

\section{REFERENCES}

[1] Malamed SF. Local anesthetics: Dentistry's most important drugs, clinical update 2006. J Calif Dent Assoc 2006;34(12):971-6.
[2] Ciechanowicz SJ, Patil VK, Association of Anaesthetists of Great Britain and Ireland. Intravenous lipid emulsion rescued at LAST. Br Dent J 2012;212(5):237-41.

[3] Weinberg GL, Laurito CE, Geldner P, et al. Malignant ventricular dysrhythmias in a patient with isovaleric acidemia receiving general and local anesthesia for suction lipectomy. J Clin Anesth 1997;9(8):668-70.

[4] Weinberg GL, VadeBoncouer T, Ramaraju GA, et al. Pretreatment or resuscitation with a lipid infusion shifts the dose - response to bupivacaine - induced asystole in rats. Anesthesiology 1998;88(4):1071-5.

[5] Weinberg GL, Palmer JW, VadeBoncouer TR, et al. Bupivacaine inhibits acylcarnitine exchange in cardiac mitochondria. Anesthesiology 2000;92(2):523-8.

[6] Rosenblatt MA, Abel M, Fischer GW, et al. Successful use of a $20 \%$ lipid emulsion to resuscitate a patient after a presumed bupivacaine-related cardiac arrest. Anesthesiology 2006;105(1):217-8.

[7] Moitra VK, Gabrielli A, Maccioli GA, et al. Anesthesia advanced circulatory life support. Can J Anaesth 2012;59(6):586-603.

[8] Neal JM, Bernards CM, Butterworth JF 4th, et al. ASRA practice advisory on local anesthetic systemic toxicity. Reg Anesth Pain Med 2010;35(2):152-61.

[9] Van den Hoek TL, Morrison LJ, Shuster M, et al. Part 12: cardiac arrest in special situations: 2010 American Heart Association guidelines for Cardiopulmonary Resuscitation and Emergency Cardiovascular Care. Circulation 2010;122(18 Suppl 3):S829-61.

[10] El-Boghdadly K, Pawa A, Chin KJ. Local anesthetic systemic toxicity: current perspectives. Local and Regional Anesthesia 2018;11:35-44. 